\title{
A Nonparametric Approach to Bond Portfolio Immunization
}

\author{
Victor Lapshin $\mathbb{D}$ \\ National Research University Higher School of Economics, 101000 Moscow, Russia; vlapshin@hse.ru
}

Received: 16 October 2019; Accepted: 14 November 2019; Published: 16 November 2019

\begin{abstract}
We consider the problem of short term immunization of a bond-like obligation with respect to changes in interest rates using a portfolio of bonds. In the case that the zero-coupon yield curve belongs to a fixed low-dimensional manifold, the problem is widely known as parametric immunization. Parametric immunization seeks to make the sensitivities of the hedged portfolio price with respect to all model parameters equal to zero. However, within a popular approach of nonparametric (smoothing spline) term structure estimation, parametric hedging is not applicable right away. We present a nonparametric approach to hedging a bond-like obligation allowing for a general form of the term structure estimator with possible smoothing. We show that our approach yields the standard duration based immunization in the limit when the amount of smoothing goes to infinity. We also recover the industry best practice approach of hedging based on key rate durations as another particular case. The hedging portfolio is straightforward to calculate using only basic linear algebra operations.
\end{abstract}

Keywords: bond portfolio immunization; nonparametric hedging; interest rate term structure; smoothing splines

MSC: $91 \mathrm{G} 10$

\section{Introduction}

Hedging a bond-like obligation with a bond portfolio is a classical topic in finance, first presented in [1]. We only give several references here to sketch the research context. Fisher and Weil [2] introduced the duration based immunization against parallel shifts in the term structure of interest rates. Cooper [3] proposed to use a parametric function to estimate the term structure and considered partial derivatives with respect to function parameters; however, his choice of parametrization was not particularly helpful. Other authors [4-6] used the Nelson-Siegel parametric model [7] to estimate the term structure and considered partial derivatives with respect to Nelson-Siegel parameters. Almeida and Lund [8] considered a richer parametric model for the term structure. Barber and Copper [9] estimated the dominant term structure change patterns via principal component analysis for the purpose of bond portfolio immunization.

Another branch of research, which is non-parametric by nature, started with Fong and Vasicek [10] and was continued by Nawalkha et al. [11,12]. They considered a Taylor approximation to the change in the portfolio price in terms of the interest rate term structure changes.

Research in this area has been concentrated on testing and comparing various methods in various settings since then.

In contrast to developed markets where it is customary to estimate interest rate term structure from derivative instruments such as interest rate swaps (IRS) or overnight indexed swaps (OIS), in many developing markets, these instruments are unavailable or relatively illiquid. This leaves government bonds as the only viable source of interest rate term structure information. Furthermore, 
even in developed markets, bonds can have much longer maturities than swaps. Finally, when the obligation is itself bond-like, it makes sense to price it using the bond-implied term structure to avoid the necessity to estimate the basis between swap and bond zero-coupon rates.

This makes the term structure endogenous and the problem of hedging an obligation with bonds different from what was studied in the literature.

To overcome this problem, we adopt a different approach. We assume that the interest rate term structure for pricing the portfolio is estimated from prices of a set of benchmark bonds via a nonparametric term structure estimation method with smoothing, i.e., by solving some variational problem. We derive the necessary hedging equations from this variational problem formulation.

To gain some intuition about the result, we also investigate some particular cases, in which we recover existing hedging approaches like the conventional duration based and convexity based hedging and the key rate duration approach introduced by Ho [13].

The remainder of the paper is structured as follows. We present the problem and its mathematical formulation in Section 2. Then, we provide the solution in Section 3. Section 4 shows that several conventional immunization methods (duration based, convexity based, and key rate durations) can be considered special cases of our general approach. Section 5 concludes.

\section{The Hedging Problem}

We consider the following hedging problem. An obligation to be hedged is described by its cash flow amounts row vector $F_{0} \in \mathbb{R}^{1 \times N}$ corresponding to the cash flow moments vector $t \in \mathbb{R}^{N}$, i.e., the cash flow at time $t_{i}$ is $\left[F_{0}\right]_{i}$. The obligation is supposed to be regularly repriced by marking to model, by using a pricing operator $P V\left(F_{0} ; r\right)$, where $r \in \mathbb{R}^{N}$ is the estimated market term structure of interest rates to be defined later, i.e., a vector of market zero-coupon rates for the corresponding terms to maturity $t$. We consider a relatively short term hedge, until the next repricing. For simplicity, ignore the time related features of investing in a bond portfolio such as changes in durations, receiving cash coupon payments and bond redemptions, etc. These only matter for a long term hedge.

The pricing operator is assumed to be linear in $F_{0}$ to allow hedging. This assumption is shown to be not restrictive even for risky coupon bearing bonds [14]. Thus, the pricing operator can be represented as:

$$
P V\left(F_{0} ; r\right)=F_{0} \cdot d(r),
$$

where $d(r) \in \mathbb{R}^{N}$ is the vector of discount factors for the corresponding terms to maturity $t$. We assume that $[d(r)]_{i}$ is a function of $r_{i}$ and $t_{i}$ only; however, the exact formula depends on the interest rate compounding convention chosen. In a more general framework, the discount factor could also be a function of credit quality, liquidity, eligibility for repo operations with the centralized counterparty, etc. However, all these parameters can be assumed constant in the short run and thus not sources of additional risks. For a long term hedge, they could be important.

Example 1. Assuming continuously compounded interest rates, we get $[d(r)]_{i}=e^{-r_{i} t_{i}}$.

As for the term structure $r$, we assume that it is estimated from a set of $K$ benchmark risk-free (e.g., government) bonds with the cash flow matrix $F \in \mathbb{R}^{K \times N}$, where $F_{k, i}$ is the cash flow for bond $k$ at term $t_{i}$. Note that the vector of payment terms $t$ can be considered common for the obligation and all the benchmark bonds. We can always introduce zero cash flows where necessary without any loss of generality.

The estimation procedure is assumed to be nonparametric, fitting the benchmark bond model prices $P V(F ; r) \in \mathbb{R}^{K}$ to their observed market prices $P \in \mathbb{R}^{K}$ while requiring some sort of smoothness as follows: 


$$
\|P V(F ; r(\cdot))-P\|^{2}+\alpha \cdot\|E[r]\|_{H^{\prime}}^{2} \rightarrow \min _{r(\cdot) \in H^{\prime}}
$$

where $r(\cdot)$ is a function to be estimated belonging to some functional space $H$, e.g., Sobolev-Hilbert space $H^{1}[0, T], E$ is a linear functional from $H$ to some $H^{\prime}$, and $\alpha$ is the smoothing (regularization) parameter. The most popular nonparametric methods of estimating the term structure of interest rates assume problems of this form [15-18].

Example 2. Two common choices for $E$ are the differentiation operator $D$ (with $H$ being $H^{1}[0, T]$ ) and $D^{2}$ (with $H=H^{2}[0, T]$ ); however, more elaborate choices might be appropriate in developing markets, as discussed in [19]. It is well known that D yields a piecewise linear term structure, while $D^{2}$ yields a smoothing cubic spline as solutions of (2).

A hedging portfolio is chosen from among the same set of benchmark bonds; let $w \in \mathbb{R}^{K}$ be the corresponding investment amounts. To simplify the exposition, we assume no trading restrictions.

The hedging problem can be formulated as follows. Given $F_{0}, P, F$, find $w$ so that the sensitivities of the hedged obligation price to price changes should be zero, which corresponds to minimizing the risk associated with the zero-coupon yield curve changes:

$$
\frac{\partial P V\left(-F_{0}+w^{T} F ; r\right)}{\partial P}=0 .
$$

Here and in what follows, we use the notation $A^{T}$ for the transpose of $A, A^{+}$for the Moore-Penrose inverse of $A, \operatorname{ker} A$ for the null space of $A, \operatorname{im} A$ for the range of $A, V^{\perp}$ for the orthogonal complement of the subspace $V$, and $\mathcal{P}_{V} A$ for the orthogonal projection of $A$ onto the subspace $V$.

\section{Results}

We now present the main result regarding the solution of the hedging problem (3). It gives the explicit expression for the hedging coefficients $w$.

Theorem 1. The hedging coefficients $w$ can be found via weighted least squares regression by regressing the vector of the obligation point sensitivities $B_{0}=\left(\frac{\partial P V\left(F_{0} ; r\right)}{\partial r}\right)^{T}$ onto the corresponding point sensitivities of the benchmark bonds $B=\left(\frac{\partial P V(F ; r)}{\partial r}\right)^{T}$ :

$$
B_{0}=B w+\varepsilon
$$

with the error covariance matrix of e equal to $\Omega=B B^{T}+A+\alpha J^{T} J$, where:

$$
A=\sum_{i=1}^{K}\left(P V\left(F_{i} ; r\right)-P_{i}\right) \frac{\partial^{2} P V\left(F_{i} ; r\right)}{\partial r^{2}}, \quad J^{T} J=\frac{1}{2} \frac{\partial^{2} G(r)}{\partial r^{2}}, \quad G(r)=\min _{f \in H \mid f\left(t_{i}\right)=r_{i}}\|E[f]\|_{H^{\prime}}^{2} .
$$

Alternatively, the hedging coefficients $w$ solve $M^{T} B w=M^{T} B_{0}$, where columns of the matrix $M=\Omega^{-1} B$ are the elementary term structure perturbations against which we hedge.

Note that the covariance matrix $\Omega$ is not singular if the solution of (2) is unique; this will be proven later. We cannot guarantee that this is indeed the case as it depends on the choice of the regularization functional $E[f]$; but since the overall idea of regularization includes guaranteeing the uniqueness of the solution, this can be safely assumed. We also assume that $F$ has full rank, which means that benchmark bond payment schedules are linearly independent. 
Example 3. For $E=D$, it is always not singular because ker $D$ only consists of constant functions $r(t)=r$, and $\left\|B^{T} r\right\|^{2}=\left\|F d^{\prime}(r) r\right\|^{2} \neq 0$ for constant $r>0$, where $d^{\prime}(r)=\frac{\partial d(r)}{\partial r}$ is a diagonal matrix. For the continuously compounded interest rates, $d^{\prime}(r)$ is given by $\left[d^{\prime}(r)\right]_{i, i}=-t_{i} e^{-r_{i} t_{i}}$.

Proof of Theorem 1. Note that even though the problem (2) is infinite-dimensional, the first term only depends on the finite-dimensional projection of $r(\cdot)$, namely on the values $r\left(t_{i}\right)$. Therefore, we can consider the following hierarchical optimization problem:

$$
\min _{r \in \mathbb{R}^{N}}\left[\min _{f(\cdot) \in H \mid f\left(t_{i}\right)=r_{i}}\left(\|P V(F ; r)-P\|^{2}+\alpha \cdot E[f]\right)\right]
$$

which can be written in a more compact way as:

$$
\|P V(F ; r)-P\|^{2}+\alpha \cdot G(r) \rightarrow \min _{r \in \mathbb{R}^{N^{\prime}}}
$$

where $r$ is a vector of zero-coupon rates at corresponding terms $t$, and $G(r)=\min _{f \in H \mid f\left(t_{i}\right)=r_{i}}\|E[f]\|_{H^{\prime}}^{2}$.

Example 4. When $E[f]=D f=f^{\prime}$, the optimal $f$ is actually a piecewise linear function connecting the known values $r_{i}$ at points $t_{i}$; therefore, $G(r)=\sum_{i=1}^{N} \frac{\left(r_{i}-r_{i-1}\right)^{2}}{t_{i}-t_{i-1}}$. Similar reasoning applies for higher derivatives with $G(r)$ also being a quadratic form in $r$.

A first order condition for an extremum in (7) is:

$$
2(P V(F ; r)-P)^{T} \frac{\partial P V(F ; r)}{\partial r}+\alpha \frac{\partial G(r)}{\partial r}=0 .
$$

Now, differentiate this with respect to $P$ :

$$
\begin{gathered}
2\left(\frac{\partial P V(F ; r)}{\partial r}\right)^{T}\left(\frac{\partial P V(F ; r)}{\partial r} \frac{\partial r}{\partial P}-I\right)+2 \sum_{i=1}^{K}\left(P V\left(F_{i} ; r\right)-P_{i}\right) \frac{\partial^{2} P V\left(F_{i} ; r\right)}{\partial r^{2}} \frac{\partial r}{\partial P}+\alpha \frac{\partial^{2} G(r)}{\partial r^{2}} \frac{\partial r}{\partial P}=0 \\
2 B\left(B^{T} \frac{\partial r}{\partial P}-I\right)+\left(2 A+2 \alpha J^{T} J\right) \frac{\partial r}{\partial P}=0
\end{gathered}
$$

where:

$$
B^{T}=\frac{\partial P V(F ; r)}{\partial r}=F \cdot d^{\prime}(r), \quad A=\sum_{i=1}^{K}\left(P V\left(F_{i} ; r\right)-P_{i}\right) \frac{\partial^{2} P V\left(F_{i} ; r\right)}{\partial r^{2}}, \quad J^{T} J=\frac{1}{2} \frac{\partial^{2} G(r)}{\partial r^{2}}
$$

Therefore,

$$
\frac{\partial r}{\partial P}=\left[B B^{T}+A+\alpha J^{T} J\right]^{-1} B=\Omega^{-1} B
$$

for $\Omega=B B^{T}+A+\alpha J^{T} J$.

Let us prove that $\Omega$ is not singular, i.e., $\operatorname{ker} J \cap \operatorname{ker}\left(B B^{T}+A\right)=\{0\}$. Assume that this is not the case and there is a nonzero $f \in \operatorname{ker} J \cap \operatorname{ker}\left(B B^{T}+A\right)$. Note that $f \in \operatorname{ker} J$ would mean that the regularization term $G(r)$ is invariant to the perturbation by $f: G(r+f)=G(r)$ for any $r$. Furthermore, $f \in \operatorname{ker}\left(B B^{T}+A\right)$ would mean that $f_{i}=0$ for every $i$ for which there is nonzero cash flow for at least one benchmark bond (this easily follows from the structure of $A$ ), which in turn implies that $P V(F ; r+f)=P V(F ; r)$. Thus, adding $f$ to the optimal solution $r^{*}$ of $(7)$ would not change the functional as $P V\left(F ; r^{*}+f\right)=P V\left(F ; r^{*}\right)$ and $G\left(r^{*}+f\right)=G\left(r^{*}\right)$. Therefore, the existence of such $f$ immediately implies the non-uniqueness of the solution of (7) or equivalently of the solution of (2), which we assumed to be unique. 
In what follows, we denote the matrix $\Omega^{-1} B$ as $M$ or $M_{\alpha}$ if the dependence on $\alpha$ is important. Using the linearity of the pricing operator, we can rewrite (3) as:

$$
\frac{\partial P V\left(F_{0} ; r\right)}{\partial P}=w^{T} \frac{\partial P V(F ; r)}{\partial P}=w^{T} B^{T} \frac{\partial r}{\partial P} ;
$$

or in terms of $M$,

$$
M^{T} B_{0}=M^{T} B w
$$

In what follows, we assume that $F$ has full row rank, i.e., there are no bonds with linearly dependent payoff schedules. This is always the case in practice. Then, $\frac{\partial P V(F ; r)}{\partial P}$ is invertible because $\Omega$ has full rank, $B=d^{\prime}(r) F^{T}$, where $d^{\prime}(r)$ is diagonal and not singular regardless of the chosen discounting convention.

Therefore, we can solve for $w$ :

$$
w=\left(\frac{\partial P V(F ; r)}{\partial P}\right)^{-T}\left(\frac{\partial P V\left(F_{0} ; r\right)}{\partial P}\right)^{T}=\left(B^{T} \Omega^{-1} B\right)^{-1} B^{T} \Omega^{-1} B_{0},
$$

where $B_{0}=\left(\frac{\partial P V\left(F_{0} ; r\right)}{\partial r}\right)^{T}$.

The expression (15) is exactly the weighted least squares estimator for the linear regression:

$$
B_{0}=B w+\varepsilon
$$

with the error covariance matrix of $\varepsilon$ given by $\Omega$ [20].

Note that the elements of $B_{0}$ and $B$ can be thought of as point sensitivities of the corresponding bond prices with respect to the spot rate $r_{i}$ for the respective term to maturity $t_{i}$ :

$$
\left[B_{0}\right]_{i}=\frac{\partial P V\left(F_{0} ; r\right)}{\partial r_{i}} .
$$

Thus, the hedging coefficients can be found by regressing the vector of the obligation point sensitivities $B_{0}$ onto the corresponding point sensitivities of the benchmark bonds $B$. The error covariance matrix depends on both the set of benchmark bonds and the smoothing functional.

As we show in Section 4, this result can be seen as a generalization of duration based, convexity based, and key rate duration hedging.

\section{Special Cases}

To develop some intuition about the solution, we now consider two special cases, as the amount of smoothing $\alpha$ goes to zero and to infinity. We show that in these special cases, we can recover the conventional duration based and convexity based immunization approaches, as well as key rate duration hedging.

\subsection{Infinite Smoothing: $\alpha \rightarrow \infty$}

First, let us show that with a suitable choice of regularization functional $E[f]$, we can recover conventional duration based and convexity based approaches.

Theorem 2. Let $w_{\alpha}$ be the vector of hedging coefficients for the smoothing parameter $\alpha$. Then:

$$
\lim _{\alpha \rightarrow \infty} w_{\alpha}=w_{\infty}
$$

where $w_{\infty}$ satisfies:

$$
\varepsilon_{\infty}=B_{0}-B w_{\infty} \in \operatorname{ker} J .
$$


Example 5. If $E[f]=D f$ is the first derivative operator, then the hedging coefficients $w_{\infty}$ provide a duration based hedge assuming continuous compounding, i.e.,

$$
\operatorname{Dur}_{0}=\operatorname{Dur} \cdot w_{\infty}
$$

where:

$$
\operatorname{Dur}_{0}=\left.\sum_{i=1}^{N} \frac{\partial P V\left(F_{0} ; r\right)}{\partial r_{i}}\right|_{r=r_{\infty}}=P V\left(F_{0} ; r_{\infty}\right)\left(\sum_{i=1}^{N} t_{i} \cdot \frac{\left[F_{0}\right]_{i} e^{-t_{i} r_{\infty}}}{P V\left(F_{0} ; r_{\infty}\right)}\right)
$$

is the dollar Fisher-Weil duration of the obligation and Dur is the row vector of the corresponding benchmark bond dollar Fisher-Weil durations.

Example 6. If $E[f]=D^{2} f$ is the second derivative operator, then the hedging coefficients $w_{\infty}$ provide a hedge based on duration and convexity assuming continuous compounding, i.e.,

$$
\operatorname{Dur}_{0}=\operatorname{Dur} \cdot w_{\infty}, C_{0}=C \cdot w_{\infty}
$$

where in addition:

$$
C_{0}=\left.\sum_{i=1}^{N} \frac{\partial^{2} P V\left(F_{0} ; r\right)}{\partial r_{i}{ }^{2}}\right|_{r=r_{\infty}}=P V\left(F_{0} ; r_{\infty}\right)\left(\sum_{i=1}^{N} t_{i}^{2} \cdot \frac{\left[F_{0}\right]_{i} e^{-t_{i} r_{\infty}}}{P V\left(F_{0} ; r_{\infty}\right)}\right)
$$

is the dollar convexity of the obligation and $C$ is the row vector of the corresponding bond dollar convexities.

Proof. It is known [21] that for any matrices $M$ and $L$ of suitable dimensions,

$$
\lim _{\alpha \rightarrow \infty}\left[M^{T} M+\alpha L^{T} L\right]^{+} M=\left[M \mathcal{P}_{\operatorname{ker} L}\right]^{+},
$$

where $\mathcal{P}_{\text {ker } L}=I-L^{+} L$ is the orthogonal projector onto ker $L$. However, in the expression for $\Omega$ (12), we have an additional convexity term $A$ inside the brackets, which is symmetric, but not nonnegative definite; thus, a separate proof is in order.

Factor $B B^{T}+A$ onto ker $J \otimes i m J^{T}$ :

$$
B B^{T}+A=\mathcal{P}_{\text {ker } J}\left(B B^{T}+A\right)+\mathcal{P}_{\operatorname{im} J^{T}}\left(B B^{T}+A\right)=\mathcal{P}_{\text {ker } J}\left(B B^{T}+A\right)+J^{T} X .
$$

Now, let $\Omega=V^{T}+U^{T}$, where $V=\left(B B^{T}+A\right) \mathcal{P}_{\text {ker } J}, U=\left(\alpha J^{T}+X^{T}\right) J$ and $U V^{T}=0$. Therefore, by Theorem 2 of [22],

$$
\Omega^{-1}=(U+V)^{-T}=\left\{U^{+}+\left(I-U^{+} V\right)\left[C^{+}+\left(I-C^{+} C\right) K V^{T} U^{+T} U^{+}\left(I-V C^{+}\right)\right]\right\}^{T},
$$

where $C=\left(I-U U^{+}\right) V$ and $K=\left[I+\left(I-C^{+} C\right) V^{T} U^{+T} U^{+}\left(I-C^{+} C\right)\right]^{-1}$. Note that $\lim _{\alpha \rightarrow \infty} U^{+}=0$ and $\lim _{\alpha \rightarrow \infty} U^{+} V=0$, both because $J$ has full column rank and thus ker $U=$ ker $J$. Therefore, $\lim _{\alpha \rightarrow \infty} K=I$ and:

$$
M=\lim _{\alpha \rightarrow \infty} \Omega^{-1} B=C^{+T} B=\left[\mathcal{P}_{\text {ker } U} V\right]^{+T}=\left[\mathcal{P}_{\text {ker } J}\left(B B^{T}+A\right) \mathcal{P}_{\text {ker } J}\right]^{+} B .
$$

Since ker $J \cup \operatorname{ker}\left(B B^{T}+A\right)=\{0\},\left.C^{+T}\right|_{\text {ker } J}$ has full rank as a mapping $\operatorname{ker} J \rightarrow \operatorname{ker} J$ and is zero otherwise. Therefore, $\operatorname{ker} M^{T}=\operatorname{ker} C^{+T}=(\operatorname{ker} J)^{\perp}$. we have:

Finally, (15) can be written as $M_{\alpha}^{T}\left(B w_{\alpha}-B_{0}\right)=0$, where $M_{\alpha}=\left(B B^{T}+A+\alpha J^{T} J\right)^{-1} B$. For $\alpha \rightarrow \infty$,

$$
M^{T}\left(B w-B_{0}\right)=M^{T} \varepsilon=0 .
$$


To observe the particular cases, note that for $E[f]$, $\operatorname{ker} J=\{x \mid x=k \cdot 1\}$, where 1 is a vector of ones and $k \in \mathbb{R}$. Therefore, the condition $\varepsilon \in(\operatorname{ker} J)^{\perp}$ reduces to $1^{T} \varepsilon=0$.

In terms of our regression, this means that:

$$
1^{T} B_{0}=1^{T} B w .
$$

Now,

$$
1^{T} B_{0}=\sum_{i=1}^{N} \frac{\partial P V\left(F_{0} ; r\right)}{\partial r_{i}}=\frac{\partial P V\left(F_{0} ; r+\xi \cdot 1\right)}{\partial \xi}=D_{0},
$$

exactly equal to the dollar Fisher-Weil duration of the obligation, while by the same $\operatorname{logic}, 1^{T} B$ is the row vector of dollar Fisher-Weil durations of the benchmark bonds. The condition (29) thus requires us to equate the dollar Fisher-Weil durations of the hedge and the obligation.

For $E[f]=D^{2}$, we similarly have ker $J=\{x \mid x=a \cdot t+b \cdot 1\}$. Thus, in addition to (30), we have:

$$
t^{T} B_{0}=\sum_{i=1}^{N} t_{i} \frac{\partial P V\left(F_{0} ; r\right)}{\partial r_{i}}=-\sum_{i=1}^{N} t_{i}^{2} e^{-r \cdot t_{i}}\left[F_{0}\right]_{i}=-\frac{1}{2} \sum_{i=1}^{N} \frac{\partial^{2} P V\left(F_{0} ; r+\xi \cdot 1\right)}{\partial \xi^{2}}=-\frac{1}{2} C_{0}
$$

where $C_{0}$ is the dollar Fisher-Weil convexity of the obligation, while by the same logic, $t^{T} B$ is the row vector of dollar Fisher-Weil convexities of the benchmark bonds times $-\frac{1}{2}$. The condition (29) thus requires us to equate both dollar Fisher-Weil durations and convexities of the hedge and the obligation.

\subsection{Virtually No Smoothing: $\alpha \rightarrow 0$}

Now, we establish the limit of the hedging portfolio composition as $\alpha \rightarrow 0$, which corresponds to estimating the term structure with non-smoothing splines.

Proposition 1. As $\alpha \rightarrow 0, \alpha^{-1} A \rightarrow A^{\prime}$, where $A^{\prime}$ is a constant diagonal matrix.

Proof. From (8) and from the fact that $B$ has full column rank, we have $P V(F ; r)-P=-\frac{\alpha}{2} B^{+}\left(\frac{\partial G(r)}{\partial r}\right)^{T}$. First note that it follows from (2) that $r \rightarrow r_{0}$ as $\alpha \rightarrow 0$, where:

$$
r_{0}=\arg \min _{r: P V(F ; r)=P_{0}} G(r)
$$

Then:

$$
\alpha^{-1} A=\sum_{i=1}^{K} \alpha^{-1}\left(P V\left(F_{i} ; r\right)-P_{i}\right) \frac{\partial^{2} P V\left(F_{i} ; r\right)}{\partial r^{2}} \rightarrow A_{0}=-\left.\sum_{i=1}^{K}\left(B^{+} J^{T} J r_{0}\right)_{i} \frac{\partial^{2} P V\left(F_{i} ; r\right)}{\partial r^{2}}\right|_{r=r_{0}},
$$

because the convergence can easily be shown to be uniform with respect to the vector $r$.

Thus, $A \sim \alpha$ as $\alpha \rightarrow 0$.

Theorem 3. As $\alpha \rightarrow 0$, the optimal hedging coefficients $w_{\alpha} \rightarrow w_{0}$, where:

$$
w_{0}=\left(M_{0} B\right)^{-1} M_{0} B_{0}, \quad M_{0}=\left(I-\left[\mathcal{P}_{(\mathrm{im} B)^{T}} A_{0} \mathcal{P}_{(\mathrm{im} B)^{\perp}}\right]^{+} \mathcal{P}_{(\mathrm{im} B)^{\perp}} A_{0}\right) B^{+T} .
$$

Proof. The following could be perceived as overly complicated. If we could assert that $\alpha^{-1} A+J^{T} J$ is not only symmetric, but also non-negative definite (and therefore could be represented as $L^{T} L$ ), a much simpler proof would follow right away along the lines of Section 4.3. 
To deal with the general case, we apply the same matrix inversion approach to $\Omega^{-1}$, but with:

$$
U=B B^{T}+\alpha\left(\alpha^{-1} A+J^{T} J\right) \mathcal{P}_{\operatorname{im} B}, \quad V=\alpha\left(\alpha^{-1} A+J^{T} J\right) \mathcal{P}_{(\mathrm{im} B)^{\perp}} .
$$
limit.

Note that as $\alpha \rightarrow 0, V \rightarrow 0, U \rightarrow B B^{T}$, and $U^{+} \rightarrow B^{+}$since $U$ does not change its rank in the

$$
\Omega^{-1}=(U+V)^{-1}=U^{+}+\left(I-U^{+} V\right)\left[C^{+}+\left(I-C^{+} C\right) K V^{T} U^{+T} U^{+}\left(I-V C^{+}\right)\right],
$$

where $C=\left(I-U U^{+}\right) V$ and $K=\left[I+\left(I-C^{+} C\right) V^{T} U^{+T} U^{+}\left(I-C^{+} C\right)\right]^{-1}$. One can easily see that $K \rightarrow I$, and thus, $\left(I-C^{+} C\right) K V^{T} U^{+T} U^{+}\left(I-V C^{+}\right) \rightarrow 0$. We now demonstrate that the limit of $C^{+} B$ exists.

$$
C^{+} B=\alpha^{-1}\left[\mathcal{P}_{(\mathrm{im} U)^{\perp}}\left(\alpha^{-1} A+J^{T} J\right) \mathcal{P}_{(\mathrm{im} B)^{\perp}}\right]^{+} B=\alpha^{-1}\left[\mathcal{P}_{(\mathrm{im} U)^{\perp}}\left(\alpha^{-1} A+J^{T} J\right) \mathcal{P}_{(\mathrm{im} B)^{\perp}}\right]^{+} \mathcal{P}_{(\mathrm{im} U)^{\perp}} B .
$$

Let us calculate $\mathcal{P}_{(\mathrm{im} U)^{\perp}} B$ as $\alpha \rightarrow 0$. First of all, note that since $B$ has full rank,

$$
\operatorname{im} U=\operatorname{im}\left(B B^{T}+\alpha\left(\alpha^{-1} A+J^{T} J\right) \mathcal{P}_{\operatorname{im} B}\right)==\operatorname{im}\left(\left(I+\alpha \tilde{A}\left(B B^{T}\right)\right)^{+}\left(B B^{T}\right)\right)=\operatorname{im}\left(\left(I+\alpha \tilde{A}\left(B B^{T}\right)^{+}\right) B\right)=\operatorname{im} \tilde{U},
$$

where $\tilde{A}=\left(\alpha^{-1} A+J^{T} J\right)$.

$$
\mathcal{P}_{(\mathrm{im} U)^{\perp}}=\mathcal{P}_{(\mathrm{im} \tilde{U})^{\perp}}=I-\tilde{U}\left(\tilde{U}^{T} \tilde{U}\right)^{-1} \tilde{U}^{T}=I-\left(I+\alpha \tilde{A}\left(B B^{T}\right)^{+}\right) B\left(\tilde{U}^{T} \tilde{U}\right)^{-1} B^{T}\left(I+\alpha\left(B B^{T}\right)^{+} \tilde{A}\right),
$$

where:

$$
\begin{gathered}
\left(\tilde{U}^{T} \tilde{U}\right)^{-1}=\left\{B^{T}\left(I+\alpha\left(B B^{T}\right)^{+} \tilde{A}\right)\left(I+\alpha \tilde{A}\left(B B^{T}\right)^{+}\right) B\right\}^{-1}= \\
\left\{B^{T} B+\alpha\left[B^{T}\left(B B^{T}\right)^{+} \tilde{A} B+B^{T} \tilde{A}\left(B B^{T}\right)^{+} B\right]+o(\alpha)\right\}^{-1}= \\
\left\{I-\alpha\left(B^{T} B\right)^{-1}\left[B^{T}\left(B B^{T}\right)^{+} \tilde{A} B+B^{T} \tilde{A}\left(B B^{T}\right)^{+} B\right]\right\}\left(B^{T} B\right)^{-1}+o(\alpha)= \\
\left(B^{T} B\right)^{-1}-\alpha\left(B^{T} B\right)^{-1}\left[B^{T}\left(B B^{T}\right)^{+} \tilde{A} B+B^{T} \tilde{A}\left(B B^{T}\right)^{+} B\right]\left(B^{T} B\right)^{-1}+o(\alpha) .
\end{gathered}
$$

Now, plug this into $\mathcal{P}_{(\operatorname{im} U)^{\perp}}$, and using $B\left(B^{T} B\right)^{-1} B^{T}=\mathcal{P}_{\operatorname{im} B}$ :

$$
\begin{aligned}
\mathcal{P}_{(\mathrm{im} U)^{\perp}}=I-\left\{\mathcal{P}_{\mathrm{im} B}+\alpha\left[\tilde{A}\left(B B^{T}\right)^{+} \mathcal{P}_{\mathrm{im} B}+\mathcal{P}_{\mathrm{im} B}\left(B B^{T}\right)^{+} \tilde{A}\right.\right. \\
\left.\left.-\mathcal{P}_{\mathrm{im} B} \tilde{A}\left(B B^{T}\right)^{+} \mathcal{P}_{\operatorname{im} B}-\mathcal{P}_{\operatorname{im} B}\left(B B^{T}\right)^{+} \tilde{A} \mathcal{P}_{\operatorname{im} B}\right]\right\}+o(\alpha)= \\
\mathcal{P}_{(\operatorname{im} B)^{\perp}}-\alpha\left[\mathcal{P}_{(\operatorname{im} B)^{\perp}} \tilde{A}\left(B B^{T}\right)^{+} \mathcal{P}_{\operatorname{im} B}+\mathcal{P}_{\operatorname{im} B}\left(B B^{T}\right)^{+} \tilde{A} \mathcal{P}_{(\operatorname{im} B)^{\perp}}\right]+o(\alpha) .
\end{aligned}
$$

Thus,

$$
\mathcal{P}_{(\mathrm{im} U)^{\perp}} B=-\alpha \mathcal{P}_{(\mathrm{im} B)^{\perp}} \tilde{A}\left(B B^{T}\right)^{+} B+o(\alpha)=-\alpha \mathcal{P}_{(\mathrm{im} B)^{\perp}} \tilde{A} B^{+T}+o(\alpha) .
$$

Now,

$$
\begin{aligned}
C^{+} B=-\left[\mathcal{P}_{(\mathrm{im} U)^{\perp}} \tilde{A} \mathcal{P}_{(\mathrm{im} B)^{\perp}}\right]^{+} \mathcal{P}_{(\mathrm{im} B)^{\perp}} \tilde{A} B^{+T}+o(1) & \rightarrow \\
& -\left[\mathcal{P}_{(\mathrm{im} B)^{\perp}} A_{0} \mathcal{P}_{(\mathrm{im} B)^{\perp}}\right]^{+} \mathcal{P}_{(\mathrm{im} B)^{\perp}} A_{0} B^{+T} .
\end{aligned}
$$

Finally,

$$
\begin{aligned}
M_{\alpha}=\Omega^{-1} B \rightarrow U^{+} B+\left(I-U^{+} V\right) C^{+} B & \rightarrow\left(U^{+}+C^{+}\right) B \rightarrow \\
& M_{0}=\left(I-\left[\mathcal{P}_{(\mathrm{im} B)^{T}} A_{0} \mathcal{P}_{(\mathrm{im} B)^{\perp}}\right]^{+} \mathcal{P}_{(\mathrm{im} B)^{\perp}} A_{0}\right) B^{+T}
\end{aligned}
$$


and:

$$
w_{\alpha}=\left(M_{\alpha} B\right)^{-1} M_{\alpha} B_{0} \rightarrow w_{0}=\left(M_{0} B\right)^{-1} M_{0} B_{0}
$$

\subsection{Key Rate Durations}

This result requires more simplifying assumptions and is itself rather simple. However, it is illustrative to see where the industry best practice approach of matching key rate durations is placed within our framework, to understand where it is not.

Proposition 2. Let the model term structure be estimated from known key rates $r^{*}$ at predefined key terms to maturity $t^{*}$ via linear interpolation and constant extrapolation. Then, the hedging weights $w^{*}$ satisfy:

$$
M^{T} B w^{*}=M^{T} B_{0},
$$

where the columns $m_{k}$ of the matrix M correspond to the key rate durations loadings, i.e., they are:

- $\quad$ equal to one for one key term to maturity $t_{k}^{*}$;

- equal to zero for all other key terms to maturity;

- are linearly interpolated for maturities between key terms;

- are constantly extrapolated outside key terms to maturity.

Proof. Note that $r=M r^{*}$ where $M$ is the linear interpolation and constant extrapolation operator defined above. The hedging equation becomes:

$$
\frac{\partial P V\left(F_{0} ; r\right)}{\partial r^{*}}=w^{T} \frac{\partial P V(F ; r)}{\partial r^{*}}
$$

which can in turn be simplified using $\frac{\partial r}{\partial r^{*}}=M$ to:

$$
M^{T} B w=M^{T} B_{0}
$$

We now present two lemmas characterizing the limit behavior of the optimal solution when partially relaxing the strict assumptions of Proposition 2.

Lemma 1. In the setup of Proposition 2, let the model term structure be determined via regularized curve fitting as follows:

$$
\left\|\mathcal{P}_{1}^{\prime} r-r^{*}\right\|^{2}+\alpha \cdot\|D r\|_{H^{\prime}}^{2} \rightarrow \min _{r(\cdot) \in H^{\prime}}
$$

where $D$ is the differentiation operator, $\mathcal{P}_{1}^{\prime}$ is the projection operator:

$$
\mathcal{P}_{1}^{\prime} r=\left[r\left(t_{1}^{*}\right), r\left(t_{2}^{*}\right) . . r\left(t_{K}^{*}\right)\right]^{T}
$$

and $r^{*}$ is the vector of observed market yields at key terms to maturity $t^{*}$.

Then, as $\alpha \rightarrow 0$, the hedging portfolio composition approaches the portfolio chosen via the key rate duration approach.

Proof. It is easy to see that Theorem 1 still holds with $B=\left(\frac{\partial \mathcal{P}_{1}^{\prime} r}{\partial r}\right)^{T}$ consisting only of ones and zeroes and $A=0$. In our setting, (15) can be written as:

$$
w_{\alpha}=\left(M_{\alpha}^{T} B\right)^{-1} M_{\alpha}^{T} B_{0}
$$


where:

$$
M_{\alpha}=\left(B B^{T}+\alpha J^{T} J\right)^{-1} B
$$

It is known that:

$$
\lim _{\alpha \rightarrow 0}\left((M K)^{T}(M K)+\alpha L^{T} L\right)^{-1}(M K)^{T} M=K_{M, L^{\prime}}^{+}
$$

where $K_{M, L}^{+}=\left(I-(L P)^{+} L\right)(M K)^{+} M$ is the $M, L$-weighted generalized pseudoinverse of $K$ and $P=I-(M K)^{+}(M K)$. Thus,

$$
\lim _{\alpha \rightarrow 0} M_{\alpha}=M_{0}=\left(I-(J P)^{+} J\right) B^{+T}=\left(I-(J P)^{+} J\right) B, \text { where } P=I-B B^{T},
$$

because $B^{+}=B^{T}$.

Let $r_{\alpha}$ be the optimal solution to (49). Then, $\frac{\partial r_{\alpha}}{\partial r^{*}}=M_{\alpha} . M_{\alpha} \rightarrow M_{0}$ and $w_{\alpha} \rightarrow w_{0}=\left(M_{0}^{T} B\right)^{-1} M_{0}^{T} B_{0}$ uniformly over $r^{*}$ as $M_{\alpha}$ does not depend on $r^{*}$ at all. Obviously, $r_{0}=\lim _{\alpha \rightarrow \infty} r_{\alpha}=M r^{*}$; the linearly interpolated key yields, so $\frac{\partial r_{0}}{\partial r^{*}}=M$. Therefore, $M_{0}=M$, and $w_{0}$ coincides with the optimal hedging weight $w$ from Proposition 2.

Lemma 2. Let the term structure be estimated from:

$$
\left\|Y(r)-r^{*}\right\|^{2}+\alpha \cdot\|D r\|_{H^{\prime}}^{2} \rightarrow \min _{r(\cdot) \in H^{\prime}}
$$

where $Y(r)$ is the vector of benchmark bond yields to maturity. Furthermore, let the benchmark bonds be coupon-bearing with coupon rates uniformly decreasing towards zero and terms to maturity $t^{*}$. If in addition, $\alpha \rightarrow 0$, then the hedging portfolio composition approaches the portfolio chosen via the key rate duration approach.

Proof. Theorem 1 holds for this case with $B=\left(\frac{\partial Y(r)}{\partial r}\right)^{T}$ and:

$$
A=\sum_{i=1}^{K}\left(Y_{i}(r)-r_{i}^{*}\right) \frac{\partial^{2} Y(r)}{\partial r^{2}} .
$$

First, let us calculate $B$.

$$
\frac{\partial Y_{i}(r)}{\partial r}=\left(\frac{\partial P V\left(F_{i} ; y \cdot 1\right)}{\partial y}\right)^{-1} \frac{\partial P V\left(F_{i} ; r\right)}{\partial r}=\frac{1}{\delta_{i}} F_{i} d^{\prime}(r)
$$

where:

$$
\delta_{i}=\frac{\partial P V\left(F_{i} ; y \cdot 1\right)}{\partial y}=\sum_{j=1}^{N} F_{i, j} d^{\prime}(y \cdot 1)_{j, j} .
$$

Note that as the coupon rate of the benchmark bonds approaches zero, $F_{i, t_{i}} \rightarrow 1$ and all other $F_{i, j} \rightarrow 0$, where $i^{*}$ is the index of the $i$ th bond term to maturity $t_{i^{*}}=t_{i}^{*}$.

Since at the same time $Y_{i}(r) \rightarrow r_{i^{*}}$, as the coupon rate decreases to zero, $\frac{\partial Y_{i}(r)}{\partial r} \rightarrow e_{i^{*}}$, where $e_{i^{*}}$ is the corresponding unit vector with one at the coordinate $i^{*}$ and zero everywhere else.

Now, let us calculate $A$.

$$
\begin{array}{r}
\frac{\partial^{2} Y_{i}(r)}{\partial r^{2}}=-\frac{1}{\delta_{i}^{2}} \frac{\partial \delta_{i}}{\partial y}\left(F_{i} d^{\prime}(r)\right)^{T} \frac{\partial y}{\partial r}+\frac{1}{\delta_{i}} F_{i} d^{\prime \prime}(r)=\frac{1}{\delta_{i}}\left(F_{i} d^{\prime \prime}(r)-\frac{1}{\delta_{i}^{2}} \frac{\partial \delta_{i}}{\partial y}\left(F_{i} d^{\prime}(r)\right)^{T}\left(F_{i} d^{\prime}(r)\right)\right)= \\
\frac{1}{\delta_{i}}\left(F_{i} d^{\prime \prime}(r)-\frac{1}{\delta_{i}^{2}}\left(\sum_{j=1}^{N} F_{i, j} d^{\prime \prime}(y \cdot 1)_{j, j}\right) d^{\prime}(r) F_{i}^{T} F_{i} d^{\prime}(r)\right) .
\end{array}
$$


Using the same logic, we get that as the coupon rate of the benchmark bonds decreases to zero, $\frac{\partial^{2} Y_{i}(r)}{\partial r^{2}} \rightarrow 0$, which means $A \rightarrow 0$.

Finally, to get the joint convergence, observe that the convergences above is uniform in $\alpha$ since it only depends on $r$ and $r \rightarrow r_{0}$ as $\alpha \rightarrow 0$.

\section{Conclusions}

The de facto industry standard of immunizing a bond-like obligation with a portfolio of bonds is based on key rate durations. It is nonparametric in its nature, but relies on having an exogenous reference term structure of interest rates determined via piecewise linear interpolation from a set of observed benchmark zero-coupon rates (e.g., interest rate swap rates).

We presented a general treatment of the immunization problem in a usual setting for developing markets, when the reference term structure of interest rates is endogenous, i.e., estimated via a smoothing spline approach from the same set of bonds (usually government bonds), which is being used for hedging.

We showed that this framework was general enough to contain both the standard duration based (Example 5) and convexity based (Example 6) hedges, as well as the key rate duration approach (Proposition 2 with the subsequent lemmas) as special cases.

This could give rise to more general practical implementations of bond portfolio immunization in developing markets. Our model was limited to short term time frames (no time effects and no changes in bond payment schedule), risk-free bonds, and perfect frictionless markets, so future research is desired to introduce the effects of time, heterogeneous credit quality, liquidity, and market frictions.

The practical implications of this work for developing markets are as follows. In the relatively widely spread case of estimating the term structure via linear interpolation of benchmark bonds' yields to maturity, the key rate duration approach could be more or less justified if the coupon rates of the benchmark bonds are small (with the key rates being equal to the benchmark bonds' terms to maturity). However, for coupon-bearing bonds or for higher orders of smoothing, this is generally not the case, and a specially designed immunization approach as described in this paper could be more desirable.

Funding: This research received no external funding.

Conflicts of Interest: The author declares no conflict of interest.

\section{References}

1. Redington, F.M. Review of the Principles of Life-office Valuations. J. Inst. Actuar. 1952, 78, $286-340$. [CrossRef]

2. Fisher, L.; Weil, R.L. Coping with the risk of interest-rate fluctuations: returns to bondholders from naive and optimal strategies. J. Bus. 1971, 44, 408-431. [CrossRef]

3. Cooper, I. Asset values, interest-rate changes, and duration. J. Financ. Quant. Anal. 1977, 12, 701-723. [CrossRef]

4. Willner, R. A new tool for portfolio managers: Level, slope, and curvature durations. J. Fixed Income 1996, 6, 48-59. [CrossRef]

5. Bravo, J. Parametric interest rate risk immunization. In New Developments in Banking and Finance; Nova Science Publishers, Inc.: New York, NY, USA, 2007; pp. 35-64.

6. Bravo, J.; Fonseca, J. Parametric Immunization in Bond Portfolio Management. In Proceedings of the 9th AFE International Conference on Applied Financial Economics, Samos Island, Greece, 28-30 June 2012.

7. Nelson, C.R.; Siegel, A.F. Parsimonious Modeling of Yield Curves. J. Bus. 1987, 60, 473-489. [CrossRef]

8. Almeida, C.; Lund, B. Immunization of fixed-income portfolios using an exponential parametric model. Braz. Rev. Econom. 2014, 34, 155-201.

9. Barber, J.R.; Copper, M.L. Immunization using principal component analysis. J. Portf. Manag. 1996, 23, 99-105. [CrossRef]

10. Fong, H.G.; Vasicek, O.A. A risk minimizing strategy for portfolio immunization. J. Financ. 1984, 39, 1541-1546. [CrossRef] 
11. Nawalkha, S.K.; Chambers, D.R. The M-Vector Model: Derivation and Testing of Extensions to M-Square. Available online: https:/ / ssrn.com/abstract=979600 (accessed on 14 November 2019).

12. Nawalkha, S.K.; Soto, G.M.; Zhang, J. Generalized M-vector models for hedging interest rate risk. J. Bank. Financ. 2003, 27, 1581-1604. [CrossRef]

13. Ho, T.S. Key rate durations: Measures of interest rate risks. J. Fixed Income 1992, 2, 29-44. [CrossRef]

14. Jarrow, R.A. Risky coupon bonds as a portfolio of zero-coupon bonds. Financ. Res. Lett. 2004, 1, 100-105. [CrossRef]

15. Waggoner, D.F. Spline Methods for Extracting Interest Rate Curves from Coupon Bond Prices; Federal Reserve Bank of Atlanta Working Paper 97-10; SSRN: Rochester, NY, USA, 1997.

16. Fisher, M.; Nychka, D.; Zervos, D. Fitting the Term Structure of Interest Rates with Smoothing Splines; Federal Reserve System Working Paper No. 95-1; SSRN: Rochester, NY, USA, 1997.

17. Laurini, M.P.; Moura, M. Constrained smoothing B-splines for the term structure of interest rates. Insur. Math. Econ. 2010, 46, 339-350. doi:10.1016/j.insmatheco.2009.11.008. [CrossRef]

18. Tanggaard, C. Nonparametric Smoothing of Yield Curves. Rev. Quant. Financ. Account. 1997, 9, $251-267$. [CrossRef]

19. Kaushanskiy, V.; Lapshin, V. A nonparametric method for term structure fitting with automatic smoothing. Appl. Econ. 2016, 48, 5654-5666. [CrossRef]

20. Strutz, T. Data Fitting and Uncertainty: A Practical Introduction to Weighted Least Squares and Beyond; Springer: Berlin/Heidelberg, Germany, 2011; p. 244. doi:10.1007/978-3-8348-9813-5. [CrossRef]

21. Eldén, L. A weighted pseudoinverse, generalized singular values, and constrained least squares problems. BIT 1982, 22, 487-502. doi:10.1007/BF01934412. [CrossRef]

22. Cline, R.E. Representations for the generalized inverse of sums of matrices. J. Soc. Ind. Appl. Math. Ser. Numer. Anal. 1965, 2, 99-114. [CrossRef]

(C) 2019 by the author. Licensee MDPI, Basel, Switzerland. This article is an open access article distributed under the terms and conditions of the Creative Commons Attribution (CC BY) license (http:// creativecommons.org/licenses/by/4.0/). 